\title{
Differentiation of Small Hepatic Hemangioma from Small Hepatocellular Carcinoma with Tri-Phasic Helical Computed Tomography Method
}

\author{
Muhammad Haseeb Jafar Aruj latif S Muhammad Yousaf Farooq Hafiz Muhammad Rizwan \\ Muhammad Ahmad Raza Muhammad Usama Sajid Mubashir Hassan \\ University Institute of Radiological Sciences \& Medical Imaging Technology, The University of Lahore, \\ Pakistan
}

\begin{abstract}
Background:

Hepatocellular carcinoma (HCC) ranks second amongst all causes of cancer deaths globally. It is on a rise in Pakistan and might represent the most common cancer in adult males. Among women, HCC is the 7th most common cancer and 6th most common cancer related death. In Pakistan prevalence of HCC varies from 3.7\%-16\% of malignant tumors and most common cause of HCC is viral hepatitis B, C and D related cirrhosis. Pakistan contributes significantly to global burden of hepatitis $\mathrm{C}$, which is a known risk factor for $\mathrm{HCC}$, and has one of the highest prevalence rates $(>3 \%)$ in the world.

Objective:

To find out the difference of small hemangioma from small hepatic cellular carcinoma by using tri- phasic helical Computed Tomography method.

Methodology:

In this descriptive study, among 81 patients of suspected hepatocellular carcinoma and hepatocellular hemangioma were selected with age and gender discrimination by convenient sampling, at Department of Radiology, UOL Teaching hospital Lahore Pakistan. 128 slice Computed Tomography Toshiba Aquilion machine was used.

Results:

Out of 81 patients collected with the suspicion of hepatic hemangioma and hepatocellular carcinoma, 41 were females and 40 were males who visited radiology department. It shows 50.6\% were females and males were $49.4 \%$. Out of 81 patients, 25 patients came with HHS and 31 were with HCC. $25.9 \%$ develop carcinoma on left side, $32.1 \%$ on right side, $6.2 \%$ on $\mathrm{R} / \mathrm{L}$ side and remaining $35.8 \%$ were Nill. Out of 81 patients 8 develop carcinoma on anterior, 5 on both, 3 on caudate, 5 on lateral,13 on both 4 on middle, 7 on posterior, 6 on segment eight, 1 on segment 4,1 on segment 7, 1 on segment 2 and remaining 36 sites were nill. $27.5 \%$ males develop HCC and $48.8 \%$ females develop HCC. Out of 81 patients $36.6 \%$ female patients develop HHS and $25.0 \%$ male patients develop HHS.

Conclusion:

In this study we conclude that females develop a large number of HCC and HHS than males. Hepatocellular carcinoma shows enhancement in early arterial and early washout phase while post-contrast images showing capsule-appearance which is relatively specific for HCC. On the other hand, HHS shows uniform enhancement in arterial phase and iso- or hyper-attenuating to liver parenchyma on delayed phase.
\end{abstract}

Keywords: Hepatocellular carcinoma, Hepatocellular hemangioma, Computed tomography

DOI: $10.7176 / \mathrm{JHMN} / 73-07$

Publication date: April $30^{\text {th }} 2020$

\section{INTRODUCTION}

Hepatocellular carcinoma (HCC) is the fifth most common malignancy worldwide and is responsible for more than 500000 deaths every year globally (1). In the United States, it most often occurs in patients with preexisting cirrhosis or chronic hepatitis. ${ }^{1}$ In the vast majority of cases, HCC develops on an underlying cirrhosis, although a fewcases have been reported in people without this condition. Despite recent improvement, the prognosis of HCC remains very poor as only about $10 \%$ of the patients can receive curative treatment as orthotopic liver transplantation (OLT) or surgical resection, which are unfeasible in most cases due to severe clinical deterioration at diagnosis and/or the inaccuracy of preoperative clinical evaluation and staging procedures. ${ }^{2} \mathrm{HCC}$ is estimated to cause between 250,000 and 1 million deaths annually, worldwide. The usefulness, frequency, and costeffectiveness of screening for HCC may well differ in different geographic areas or among different ethnic populations, because there may be differences in the incidence and growth characteristics of HCC. There are also likely major differences in the epidemiology of hepatitis B between endemic and non endemic areas, particularly with respect to age at which the disease is acquired. ${ }^{3}$ Hepatic hemangioma $(\mathrm{HH})$ is the most common benign hepatic tumor. The features may lead to a misdiagnosis of hepatocellular carcinoma (HCC). HCC usually occurs as a complication of chronic liver disease and most often arises in cirrhotic livers. The accurate differentiation of 
HH from HCC with or without cirrhosis is important for patient care and treatment decisions. ${ }^{4}$ High-flow hepatic hemangiomas are more likely to accompany arterioportal shunt than slow-flow hemangiomas [1]. It has been reported that histopathologic differences exist between the high-flow and the slowflow hemangiomas. ${ }^{5}$

Benign tumors of the liver include hemangiomas, focal nodular hyperplasia, and liver cell adenoma. Hepatic hemangiomas are the most common benign tumors of the liver, with an incidence in autopsy series ranging from $0.4 \%$ to $7.3 \% .1$ These lesions are considered congenital vascular malformations and enlarge by ectasia rather than neoplastic growth. Macroscopically, they are well-circumscribed, reddish-purple, hypervascular lesions that are compressible. Histologic analysis of these tumors reveals large blood-filled spaces lined by endothelial cells and separated by thin fibrous septa. ${ }^{6}$ Primary carcinoma of the liver holds a unique position among all human neoplasms: first, because of striking differences in racial and geographical distribution, the problem, has worldwide significance. Second, it is so regularly associated with a chronic degenerative and regenerative disease (cirrhosis) of a large parenchymatous organ. Such association is rarely seen in other large organs such as the pancreas and kidney. Third, tumors occur spontaneously and can also be easily produced in experimental animals by different carcinogenic agents that damage the liver. Lastly, certain inherent difficulties are encountered in both the clinical and pathological aspects of the disease. The clinical problem is often one of diagnosing carcinoma in the presence of cirrhosis or distinguishing Primary from secondary carcinoma of the liver. From the standpoint of pathology, there is such variation in histological structure that the determination of histogenesis and hence classification of some neoplasms of the liver have been almost impossible. ${ }^{7}$ Dual-energy CT performed during two consecutive scans or with a dual x-ray source, dual-detector assembly has existed for a number of years and yields additional information that is used for material separation at imaging, with potential use in clinical applications ( 5-8 ). However, the clinical use of dual-energy CT has been hampered because of motion misregistration, high image noise, and excessive radiation exposure caused by long acquisition times ( 8 ). A CT scanning mode based on the rapid switching between high- and low-energy data sets from view to view was recently introduced. This scanning mode enables precise registration of data sets for creation of accurate materialdecomposition images (eg, water- and iodine-based material-decomposition images) and monochromatic spectral images at energy levels ranging from 40 to $140 \mathrm{keV}$ throughout the full $50-\mathrm{cm}$ fi eld of view. The garnet crystal detector used, with an extremely fast primary speed (100 times faster than existing detectors) and low afterglow (four times lower), and the associated detection system enable simultaneous dual-energy acquisition. ${ }^{4}$

While the computed tomographic (CT) and magnetic resonance (MR) imaging characteristics of HCC have been documented extensively, HCC can have a variety of appearances. Many radiologists and referring physicians would probably regard any hypervascular, noncystic, focal hepatic lesion in a cirrhotic liver as being highly suggestive of HCC. In a series of 508 consecutive hepatectomy specimens obtained during liver transplantation, Dodd et al (2) found only nine cavernous hemangiomas at gross pathologic examination, and only three of these were visualized at preoperative nonhelical CT. This incidence of hemangiomas $(1.7 \%$ at pathologic examination, $0.6 \%$ at $\mathrm{CT}$ ) is lower than the frequency with which hemangiomas are encountered at unselected autopsy series (3), or at CT in noncirrhotic patients. ${ }^{8}$ Multiphasic helical computed tomographic (CT) examination of the liver following intravenous contrast material injection has become an important technique for the detection and characterization of hepatic masses. Several reports $(1-6)$ have focused on detection of liver tumors with the use of multiphasic helical CT, and it has been reported (3) that two-phase (arterial and portal venous phase) helical CT is useful in the detection of hypervascular liver tumors. However, only a few reports $(7,8)$ have focused on characterization of liver tumors with use of multiphasic helical CT. Most cavernous hemangiomas are easily distinguished from malignant hepatic tumors due to characteristic features, such as near isoattenuation with blood on nonenhanced images and globular or nodular peripheral enhancement similar to attenuation of blood vessels, at rapid CT with bolus administration of contrast material. Because hemangiomas are encountered frequently, distinction from hepatic malignancy is an important and common challenge. ${ }^{9}$

An arterioportal (AP) shunt associated with a hepatic tumor has been reported to be an important sign that the tumor is malignant (5-7), but AP shunts have been believed to be rare in hemangiomas $(5,8-10)$. However, results of more recent studies have shown that AP shunts are not uncommonly seen in hepatic hemangiomas. Several authors have reported that a high percentage of hemangiomas $(19 \%-26 \%)$ are accompanied by an AP shunt $(3,11,12)$. In contrast, most HCCs accompanied by AP shunts tend to be advanced tumors with portal vein thrombosis. To our knowledge, a comparison of the prevalence of AP shunts associated with small hemangiomas and that associated with HCCs $3 \mathrm{~cm}$ in diameter or smaller has not been performed by using two-phase helical CT except in one study (13), the results of which revealed that differential diagnosis between early and homogeneously enhancing $\mathrm{HCC}$ and hemangioma was possible with twophase CT. ${ }^{10}$

\section{Methods}

In this descriptive study, among 81 patients of suspected hepatocellular carcinoma and hepatocellular hemangioma were selected with age and gender discrimination by convenient sampling, at Department of Radiology, UOL Teaching hospital Lahore Pakistan. 128 slice Computed Tomography Toshiba Aquilion machine was used. 
Patients included in this study had a clinical evidence of liver carcinoma. Pregnant patients were excluded in this study.

\section{RESULTS}

Out of 81 patients collected with the suspicion of hepatic hemangioma and hepatocellular carcinoma.41 were females and 40 were males who visited radiology department. It shows 50.6\% were females and males were $49.4 \%$. Out of 81 patients, 25 patients came with HHS and 31 were with HCC. $25.9 \%$ develop carcinoma on left side, $32.1 \%$ on right side, $6.2 \%$ on $\mathrm{R} / \mathrm{L}$ side and remaining $35.8 \%$ were Nill. Out of 81 patients 8 develop carcinoma on anterior, 5 on both, 3 on caudate, 5 on lateral, 13 on both 4 on middle, 7 on posterior, 6 on segment eight, 1 on segment 4,1 on segment 7,1 on segment 2 and remaining 36 sites were nill. $27.5 \%$ males develop HCC and $48.8 \%$ females develop HCC. Out of 81 patients $36.6 \%$ female patients develop HHS and $25.0 \%$ male patients develop HHS.

Gender

\begin{tabular}{|ll|r|r|r|r|}
\hline & Frequency & Percent & Valid Percent & Cumulative Percent \\
\hline Valid & F & 41 & 50.6 & 50.6 & 50.6 \\
& M & 40 & 49.4 & 49.4 & 100.0 \\
& Total & 81 & 100.0 & 100.0 & \\
\hline
\end{tabular}

Table 1: Frequency distribution of gender

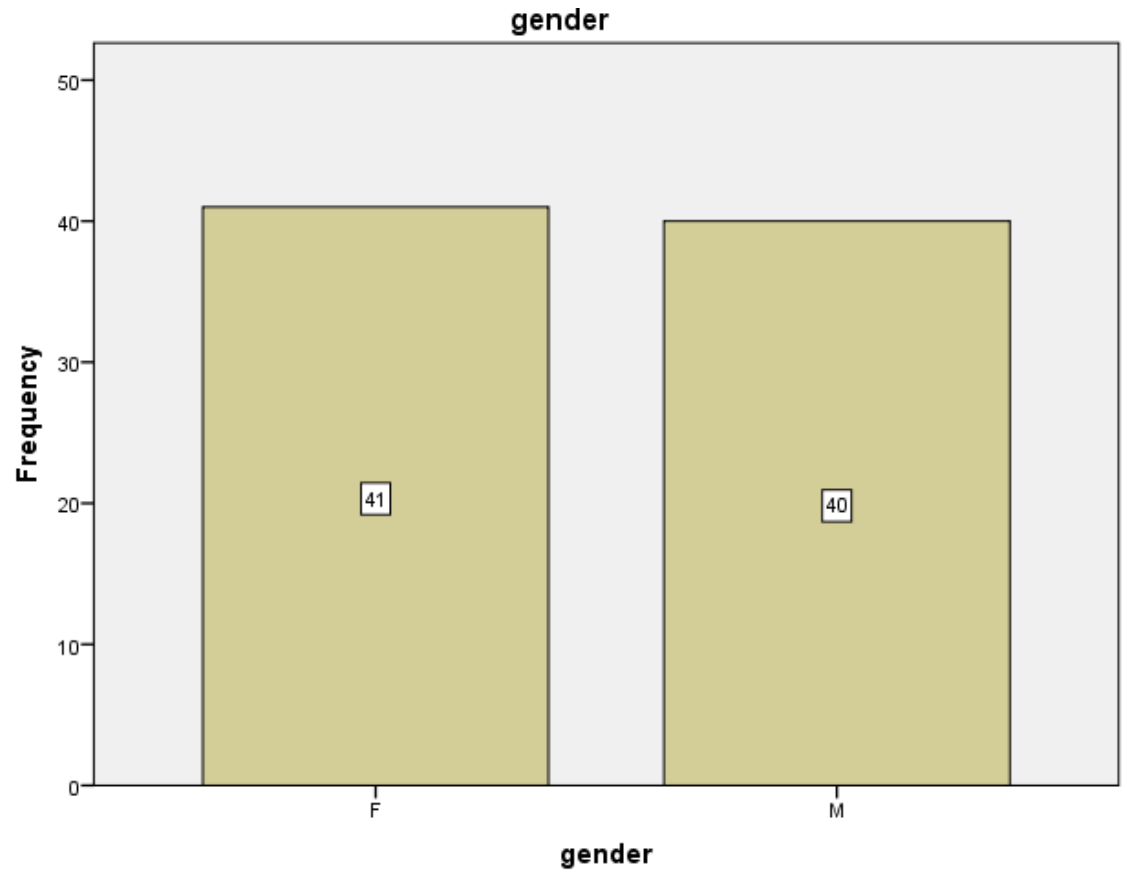

Hepatic Hemangiomas

\begin{tabular}{|ll|r|r|r|r|}
\hline & \multicolumn{2}{|c|}{ Frequency } & Percent & Valid Percent & Cumulative Percent \\
\hline Valid & NO & 56 & 69.1 & 69.1 & 69.1 \\
& YES & 25 & 30.9 & 30.9 & 100.0 \\
& Total & 81 & 100.0 & 100.0 & \\
\hline
\end{tabular}

Table 2: Frequency distribution of hepatic hemangiomas 


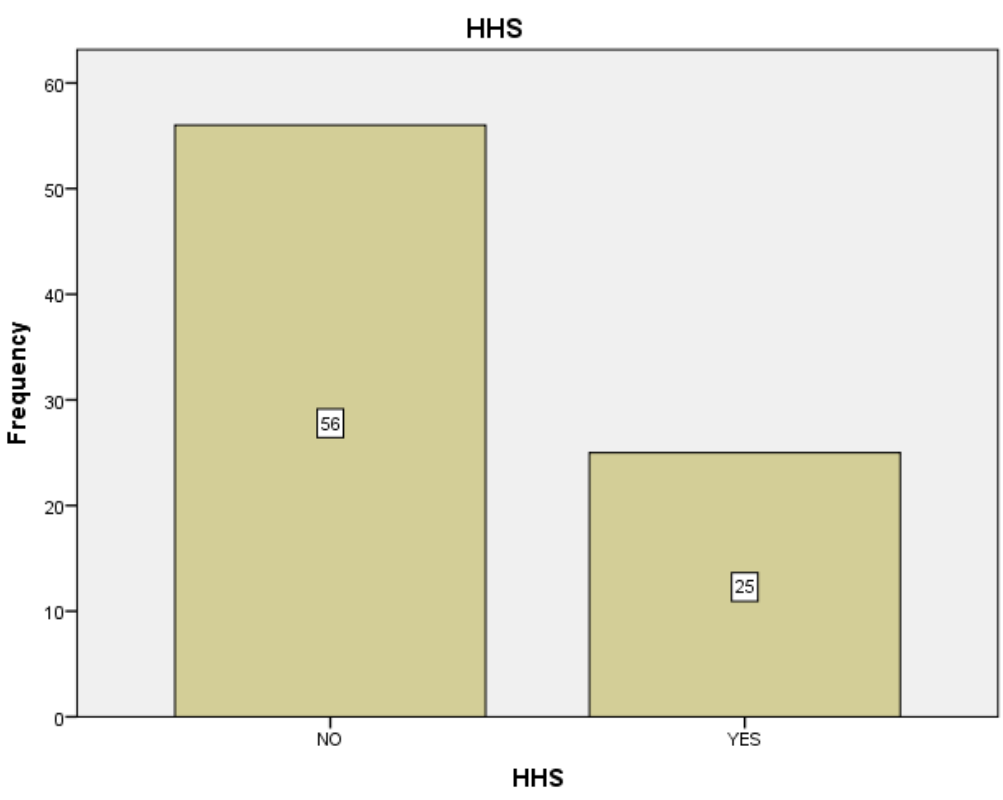

Hepatocellular Carcinomas

\begin{tabular}{|ll|r|r|r|r|}
\hline & \multicolumn{1}{|c|}{ Frequency } & \multicolumn{1}{|c|}{ Percent } & \multicolumn{1}{c|}{ Valid Percent } & Cumulative Percent \\
\hline Valid & NO & 50 & 61.7 & 61.7 & 61.7 \\
& YES & 31 & 38.3 & 38.3 & 100.0 \\
& Total & 81 & 100.0 & 100.0 & \\
\hline
\end{tabular}

Table 3: Frequency distribution of hepatocellular carcinoma

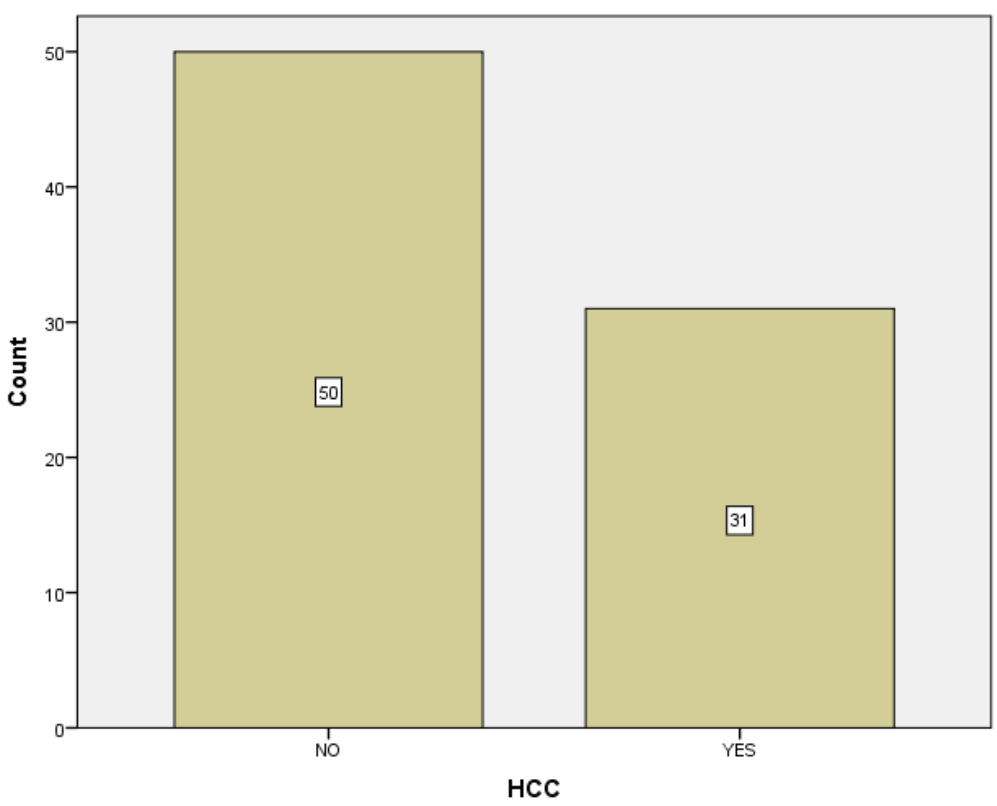

Side

\begin{tabular}{|ll|r|r|r|r|}
\hline & Frequency & Percent & Valid Percent & Cumulative Percent \\
\hline Valid & LEFT & 21 & 25.9 & 25.9 & 25.9 \\
& NILL & 29 & 35.8 & 35.8 & 61.7 \\
& RIGHT & 26 & 32.1 & 32.1 & 93.8 \\
RIGHT ,LEFT & 5 & 6.2 & 6.2 & 100.0 \\
& 81 & 100.0 & 100.0 & \\
\hline
\end{tabular}

Table 4: Frequency distribution of side 


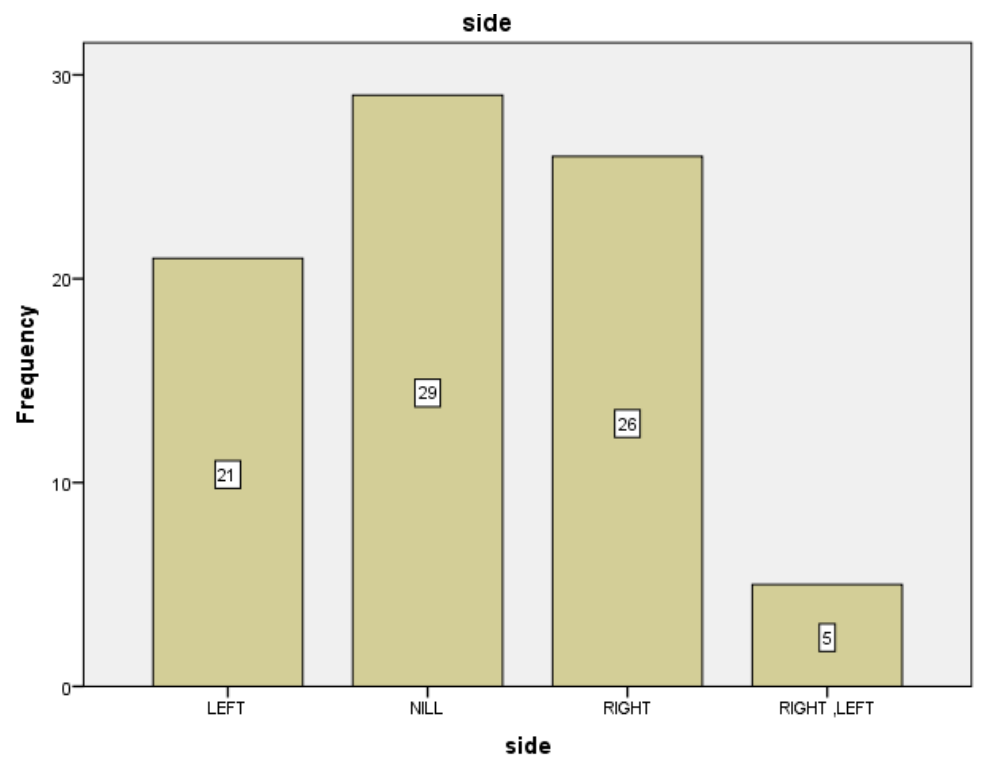

Site

\begin{tabular}{|ll|r|r|r|r|}
\hline & Frequency & Percent & Valid Percent & Cumulative Percent \\
\hline Valid & 8 & 9.9 & 9.9 & 9.9 \\
& ANTERIOR & 5 & 6.2 & 6.2 & 16.0 \\
BOTH LOBE & 3 & 3.7 & 3.7 & 19.8 \\
CAUDATE & 5 & 6.2 & 6.2 & 25.9 \\
LATERAL & 13 & 16.0 & 16.0 & 42.0 \\
LOBE & 4 & 4.9 & 4.9 & 46.9 \\
MIDDLE & 26 & 32.1 & 32.1 & 79.0 \\
NILL & 7 & 8.6 & 8.6 & 87.7 \\
POSTERIOR & 6 & 7.4 & 7.4 & 95.1 \\
SEGMENT EIGHT & 1 & 1.2 & 1.2 & 96.3 \\
SEGMENT FOUR & 1 & 1.2 & 1.2 & 97.5 \\
SEGMENT SEVEN & 1 & 1.2 & 1.2 & 98.8 \\
SEGMENT SIX & 1 & 1.2 & 1.2 & 100.0 \\
SEGMENT TWO & 81 & 100.0 & 100.0 & \\
Total & & & &
\end{tabular}

Table 5: Frequency distribution of site

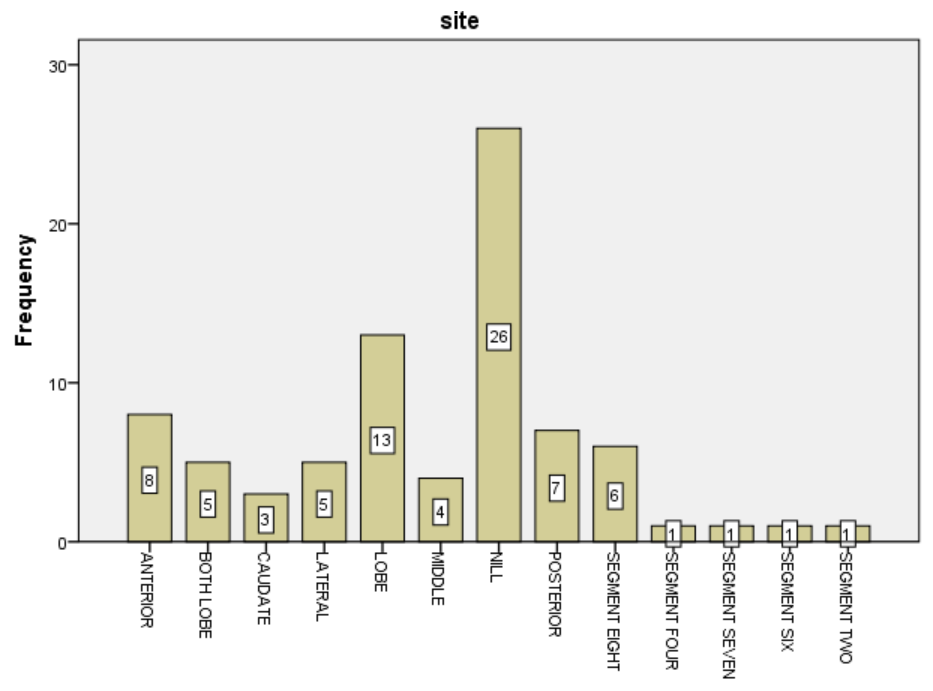


Gender * HCC Crosstabulation

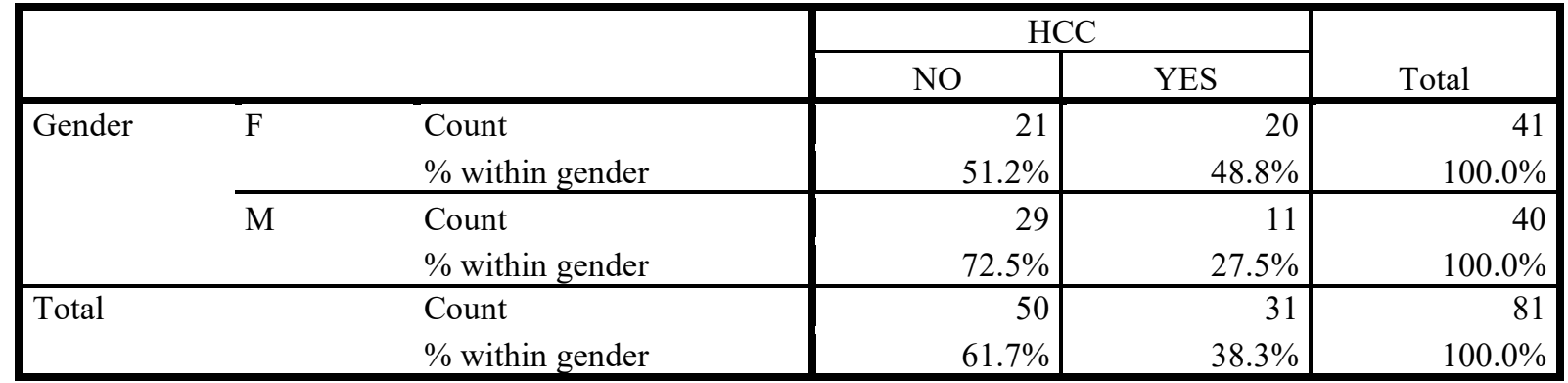

Table 5: Frequency distribution of gender * HCC Crosstabulation

Gender * HHS Crosstabulation

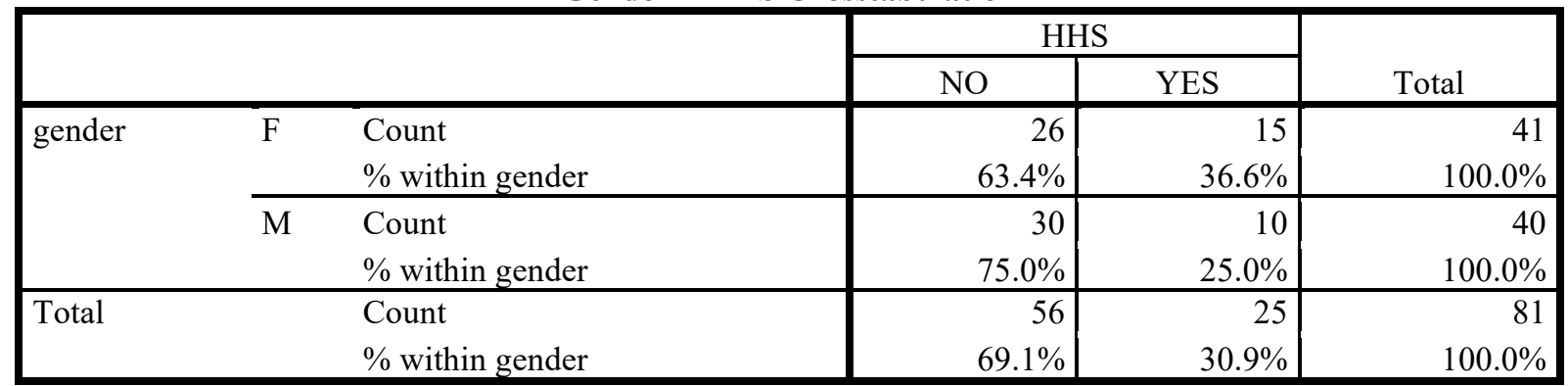

Table 5: Frequency distribution of gender * HHS Crosstabulation

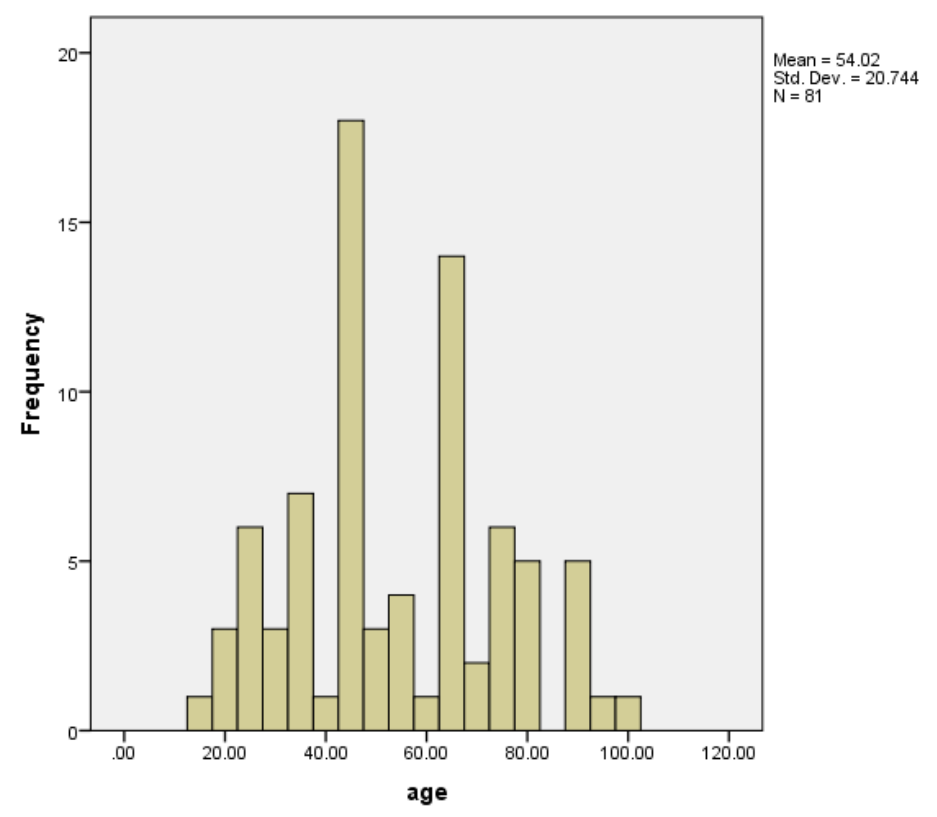

\section{Discussion}

Out of 81 patients collected with the suspicion of hepatic hemangioma and hepatocellular carcinoma. 41 were females and 40 were males who visited radiology department. It shows 50.6\% were females and males were $49.4 \%$. Out of 81 patients, 25 patients came with HHS and 31 were with HCC. $25.9 \%$ develop carcinoma on left side, $32.1 \%$ on right side, $6.2 \%$ on $\mathrm{R} / \mathrm{L}$ side and remaining $35.8 \%$ were Nill. Out of 81 patients 8 develop carcinoma on anterior, 5 on both, 3 on caudate, 5 on lateral, 13 on both 4 on middle, 7 on posterior, 6 on segment eight, 1 on segment 4, 1 on segment 7, 1 on segment 2 and remaining 36 sites were nill. $27.5 \%$ males develop HCC and $48.8 \%$ females develop HCC. Out of 81 patients $36.6 \%$ female patients develop HHS and 25.0\% male patients develop HHS. Hayashida M et.al conducted a study which results in Forty-three (67\%) of 64 lesions showed Grade 4 (n $=24)$ or Grade $5(n=19)$ enhancement on arterial-phase CT, indicating hypervascular HCCs on CT (Fig. 1). In contrast, $51(80 \%)$ of 64 lesions showed Grade $4(n=20)$ or Grade $5(n=31)$ enhancement on arterial-phase MR imaging, indicating hypervascular HCCs on MR imaging. All of the 43 hypervascular HCCs on arterial-phase CT were included in the 51 hypervascular HCCs on MR imaging. The grading score of hypervascular HCCs on 
arterial-phase MR imaging (mean: 4.61) was significantly (P b.01) higher than that of hypervascular HCCs on arterial-phase CT (mean: 4.20), showing better detection of hypervascularity (arterial enhancement) of the lesions on arterial-phase MR imaging. Thirty-two (74\%) of 43 hypervascular HCCs on CT showed Grade $1(\mathrm{n}=13)$ or Grade 2 ( $\mathrm{n}=19)$ enhancement on late-phase CT. In contrast, $25(49 \%)$ of 51 hypervascular HCCs on MR imaging showed Grade $1(n=11)$ or Grade $2(n=14)$ enhancement on late-phase MR imaging (Fig. 1). Grading scores of hypervascular HCC on late-phase CT (mean: 2.00) were significantly ( $\mathrm{P} \mathrm{b.001)} \mathrm{lower} \mathrm{than} \mathrm{those} \mathrm{on} \mathrm{MR} \mathrm{imaging}$ (mean: 2.35), indicating less washout effects for hypervascular HCCs on late-phase MR imaging. Twenty-one (33\%) of 64 lesions showed Grade 1, 2 or 3 enhancement on arterial-phase CT, indicating hypovascular HCCs on CT. All of these 21 hypovascular HCCs on CT showed Grade $1(n=10)$ or Grade $2(n=11)$ enhancement on latephase CT seen as hypoattenuation. In contrast, $13(20 \%)$ of 64 lesions showed Grade 1, 2 or 3 enhancement on arterialphase MR imaging, indicating hypovascular HCCs on MR imaging. Among these 13 hypovascular HCCs on MR imaging, $8(62 \%)$ lesions showed Grade $1(\mathrm{n}=1)$ or Grade $2(\mathrm{n}=7)$ enhancement on late-phase MR imaging, seen as hypointensity. The remaining five (38\%) hypovascular HCCs on MR imaging showed Grade 3 enhancement, seen as isointensity. Grading scores of hypovascular HCCs on latephase images were significantly (P b.001) lower on CT than on MR imaging (mean score: 1.52 vs. 2.31), indicating better washout effects for hypovascular HCCs on late-phase CT. Although five (38\%) hypovascular HCCs were not detected (Grade 3) on arterial-phase and late-phase MR imagings, all of these HCCs were visible on unenhanced T1-weighted and/or T2-weighted MR images. ${ }^{11} \mathrm{~L}$ v, P et.al concluded conventional CT methods, globular enhancement was observed in $19(63 \%)$ and $18(60 \%)$ of the $30 \mathrm{HH}$ lesions during the AP and PVP, respectively; in zero and one $(11 \%)$ of the nine HCCcirrhosis lesions during the AP and PVP, respectively; and in four (15\%) and three (12\%) of the 26 HCC-no cirrhosis lesions during the AP and PVP, respectively. Attenuation similar to that of the aorta during the AP and similar to that of the blood pool during the PVP was observed in $14(47 \%)$ of the $30 \mathrm{HH}$ lesions and in none of the HCC lesions (with or without cirrhosis). During the AP, diffuse homogeneous enhancement was noted in eight $(27 \%)$ of the $30 \mathrm{HH}$ lesions, seven (78\%) of the nine HCCcirrhosis lesions, and $13(50 \%)$ of the $26 \mathrm{HCC}-$ no cirrhosis lesions. Using these qualitative criteria, we achieved sensitivities and specifi cities of $67 \%$ and $56 \%$, respectively, for differentiating between $\mathrm{HH}$ and $\mathrm{HCC}$ with cirrhosis and $67 \%$ and $54 \%$, respectively, for differentiating between $\mathrm{HH}$ and $\mathrm{HCC}$ without cirrhosis during the AP. We achieved sensitivities and specifi cities of $83 \%$ and $78 \%$, respectively, for differentiating between $\mathrm{HH}$ and $\mathrm{HCC}$ with cirrhosis and $83 \%$ and $84 \%$, respectively, for differentiating between $\mathrm{HH}$ and $\mathrm{HCC}$ without cirrhosis during the PVP. ${ }^{4}$ Another study was conducted by Freeny PC et.al which results inthe patterns of dynamic contrast enhancement in the 58 scans were categorized as peripheral in 74\% (43 hemangiomas); central (focal zone of contrast enhancement within the center of the lesion) in 12\% (seven hemangiomas); diffuse (complete, homogeneous contrast enhancement of the entire lesion) in $2 \%$ (one hemangioma); mixed (central and peripheral) in $9 \%$ (five hemangiomas); and absent in $3 \%$ (two hemangiomas). The degree of isodense fill-in was categorized as complete in $72 \%$ (42 hemangiomas), partial (a remaining zone of diminished attenuation within the lesion) in $21 \%$ (12 hemangiomas), and none (no fill-in or decrease in lesion size) 7\% (four hemangiomas). Included in the last group were two hemangiomas in the same patient that arose in a diffusely fatty liver. The lesions remained hyperdense on delayed scans and therefore were categorized as having no fill-in. The time required to reach complete isodense fill-in was determined for each hemangioma. There was no direct relationship to size and time required to reach isodense fill-in, although the largest lesion $(12 \mathrm{~cm})$ required the longest time $(90 \mathrm{~mm})$. Only two of the 42 hemangiomas required more than 30 $\mathrm{mm}$ to reach fill-in (45 and $90 \mathrm{mi}$ respectively). During the delayed scan sequences, 13 lesions remained diffusely hyperdense for 5-15 mm before becoming isodense. All lesions that were diffusely hyperdense became isodense. In five cases, delayed scans were obtained after isodense fill-in had occurred. These scans showed that the lesions subsequently returned to their precontrast appear- ance (hypodense relative to the surrounding hepatic parenchyma). ${ }^{12}$

\section{CONCLUSION}

In this study we conclude that females develop a large number of HCC and HHS than males. Hepatocellular carcinoma shows enhancement in early arterial and early washout phase while post-contrast images showing capsule-appearance which is relatively specific for HCC. On the other hand, HHS shows uniform enhancement in arterial phase and iso- or hyper-attenuating to liver parenchyma on delayed phase.

\section{REFERENCES}

1. Sahani DV, Holalkere NS, Mueller PR, Zhu AX. Advanced hepatocellular carcinoma: CT perfusion of liver and tumor tissue - initial experience. Radiology. 2007 Jun;243(3):736-43.

2. Colli A, Fraquelli M, Casazza G, Massironi S, Colucci A, Conte D, Duca P. Accuracy of ultrasonography, spiral CT, magnetic resonance, and alpha-fetoprotein in diagnosing hepatocellular carcinoma: a systematic review. American Journal of Gastroenterology. 2006 Mar 1;101(3):513-23.

3. Sherman M, Peltekian KM, Lee C. Screening for hepatocellular carcinoma in chronic carriers of hepatitis B 
virus: incidence and prevalence of hepatocellular carcinoma in a North American urban population. Hepatology. 1995 Aug;22(2):432-8.

4. Lv P, Lin XZ, Li J, Li W, Chen K. Differentiation of small hepatic hemangioma from small hepatocellular carcinoma: recently introduced spectral CT method. Radiology. 2011 Jun;259(3):720-9.

5. Kim KW, Kim AY, Kim TK, Kim SY, Kim MJ, Park MS, Park SH, Lee KH, Kim JK, Kim PN, Ha HK. Hepatic hemangiomas with arterioportal shunt: sonographic appearances with CT and MRI correlation. American Journal of Roentgenology. 2006 Oct;187(4):W406-14.

6. Yoon SS, Charny CK, Fong Y, Jarnagin WR, Schwartz LH, Blumgart LH, DeMatteo RP. Diagnosis, management, and outcomes of 115 patients with hepatic hemangioma. Journal of the American College of Surgeons. 2003 Sep 1;197(3):392-402.

7. Edmondson HA, Steiner PE. Primary carcinoma of the liver. A study of 100 cases among 48,900 necropsies. Cancer. 1954 May;7(3):462-503.

8. Brancatelli G, Federle MP, Blachar A, Grazioli L. Hemangioma in the cirrhotic liver: diagnosis and natural history. Radiology. 2001 Apr;219(1):69-74.

9. Kim T, Federle MP, Baron RL, Peterson MS, Kawamori Y. Discrimination of small hepatic hemangiomas from hypervascular malignant tumors smaller than $3 \mathrm{~cm}$ with three-phase helical CT. Radiology. 2001 Jun;219(3):699-706.

10. Byun JH, Kim TK, Lee CW, Lee JK, Kim AY, Kim PN, Ha HK, Lee MG. Arterioportal shunt: prevalence in small hemangiomas versus that in hepatocellular carcinomas $3 \mathrm{~cm}$ or smaller at two-phase helical CT. Radiology. 2004 Aug;232(2):354-60.

11. Hayashida M, Ito K, Fujita T, Shimizu A, Sasaki K, Tanabe M, Matsunaga N. Small hepatocellular carcinomas in cirrhosis: differences in contrast enhancement effects between helical CT and MR imaging during multiphasic dynamic imaging. Magnetic resonance imaging. 2008 Jan 1;26(1):65-71.

12. Freeny PC, Marks WM. Hepatic hemangioma: dynamic bolus CT. American Journal of Roentgenology. 1986 Oct 1;147(4):711-9. 